This item was submitted to Loughborough's Research Repository by the author.

Items in Figshare are protected by copyright, with all rights reserved, unless otherwise indicated.

\title{
Are nanoparticles spherical or quasi-spherical?
}

PLEASE CITE THE PUBLISHED VERSION

http://dx.doi.org/10.1002/chem.201500807

PUBLISHER

(c) Wiley

VERSION

AM (Accepted Manuscript)

LICENCE

CC BY-NC-ND 4.0

REPOSITORY RECORD

Sokolov, Stanislav V., Christopher Batchelor-McAuley, Kristina Tschulik, Stephen Fletcher, and Richard G. Compton. 2015. “Are Nanoparticles Spherical or Quasi-spherical?”. Loughborough University. https://hdl.handle.net/2134/21060. 


\title{
Are Nanoparticles Spherical or Quasi-Spherical?
}

\author{
Stanislav V. Sokolov ${ }^{1}$, Christopher Batchelor-McAuley ${ }^{1}$, Kristina Tschulik ${ }^{1}$, Stephen \\ Fletcher ${ }^{2}$, and Richard G. Compton $*^{1}$
}

1. Department of Chemistry, Physical and Theoretical Chemistry Laboratory, Oxford University, South Parks Road, Oxford OX1 3QZ, UK

2. Department of Chemistry, Loughborough University, Leicestershire, LE11 3TU, UK

Published 20 Jul 2015. DOI: 10.1002/chem.201500807

(C) 2015 WILEY-VCH Verlag GmbH \& Co. KGaA, Weinheim

\begin{abstract}
The geometry of quasi-spherical nanoparticles is investigated. The combination of SEM imaging and electrochemical nano-impact experiments is demonstrated to allow sizing and characterization of the geometry of single silver nanoparticles.
\end{abstract}

\section{Keywords}

Nano-impacts, coulometric sizing, quasi-spherical nanoparticles, icosahedral nano-particles.

\section{Introduction}

Nanoparticles (NPs) have been synthesized in a large variety of shapes and sizes. The resultant properties can be adjusted for a given purpose and are particularly important in biomedical applications. [1,2] Traditionally, spherical nanoparticles have been the main focus of research, but recently quasi-spherical NPs have shown a range of advantageous properties.[3] Particular examples of non-spherical particles are nano-cubes, which show potential for hyperthermia treatment owing to the larger magnetic anisotropy of a cubic structure compared with a sphere.[4] In addition, quasi-spherical particles are particularly important for catalytic applications. One example is the oxygen reduction reaction (ORR), [5] which is strongly influenced by the presence of $\mathrm{Au}(100)$ surface domains on Au nanoparticles; indeed, the greatest catalytic activity is observed for particles that deviate from spherical geometry.

Accurate nanoparticle sizing is crucial, and numerous methods have been applied such as transmission electron microscopy (TEM), scanning electron microscopy (SEM), dynamic light scattering (DLS) and nanoparticle tracking analysis (NTA). The main advantage of electron microscopy [6] is that readily 
understandable visual shape information is obtained. However, the number of particles probed is generally small (<1000 particles) and careful sample preparation is required to carry out accurate measurements in vacuo. In particular, particle aggregation during drying of the sample has to be avoided as far as possible. Three-dimensional information can be obtained from TEM and SEM imaging by using a tilt-series of images, [7] but the process is time-consuming and often requires complex analysis. DLS and NTA are also routinely used as a first means of sizing NPs. DLS can provide information regarding the average size of the ensemble of nanoparticles but the resulting size distribution is typically skewed towards larger particles and may strongly depend on the instrument and analytical procedure used. [8] The sample preparation is simple and analysis is performed in solution phase. The basis of the technique is the measurement of the particle diffusion coefficient, which is then related to its hydrodynamic radius. As a result, all shape information is lost. With a correction for anisotropic diffusion, DLS has been successfully applied to nanorods, $[9,10]$ but applications to other geometries are yet to appear. NTA can provide information regarding individual particles [11] and is less affected by presence of large particles. However, the accuracy of the obtained data is strongly dependent on video capture and analysis and is strongly affected by the skill of the operator. [11]

"Nano-impacts" provide volume information for individual particles. Given that a statistically significant number of particles is analyzed, it also becomes possible to estimate the distribution of sizes. In contrast to electron microscopy imaging, nano-impacts can be performed in the solution phase, reducing the influence of aggregation. A typical experimental setup consists of a microelectrode, held at a potential that allows full oxidation or reduction of a colliding nanoparticle, leading to a spike in a chronoamperogram. The area of each spike quantifies the charge transferred to the nanoparticle and is proportional to its size.

Electrochemical nano-impacts have recently been demonstrated to yield accurate size information for a range of different nanoparticle systems (silver, [13] gold, [14] nickel, [14] iron oxide, [15] C60 carbon nanoparticles, [16] and other organic nanoparticles [17, 18]. and offer an attractive alternative to the more traditional sizing methods due to the low cost and relative simplicity of sample preparation, whilst retaining the merits of in situ analysis. Unlike imaging techniques, which typically provide a two-dimensional top view image of the particles, nano-impacts provide the number of atoms in a nanoparticle and corresponding volumetric information by the charge passed in the electrolysis of the particle.

According to the literature, there is a wide range of micrometer-sized polyhedral crystals and their formation and characterization has been investigated electrochemically [19] and through the electron microscopy; [20] however, no such analysis has been performed on the nano-scopic scale. In the present report, we introduce a general geometrical analysis for quasi-spherical nanoparticles and propose a joint technique of electron microscopy imaging and nano-impacts for accurate sizing of such particles. The application of this technique is demonstrated experimentally for icosahedral-shaped silver nanoparticles by using nanoimpacts and SEM images to show the merits of simultaneous use of the two techniques. By combining the size distributions for quasi-spherical icosahedral particles obtained through SEM imaging and nano-impacts and applying a geometric analysis, we establish an accurate description of the nanoparticle geometry and size.

\section{Theory}

A nanoparticle is characterized by its dimensions and its density. Any solid of a given geometry has a minimum set of parameters that describe it. For example, a perfect sphere is fully described by its radius; if the radius is known, additional information such as volume and surface area can be obtained immediately. For the purpose of nanoparticle sizing, a 'spherical' approximation is often used to simplify the analysis for quasi-spherical NPs. The size distributions of particles are commonly reported in terms of the spherical radius or diameter. Geometrically this is represented by a sphere touching each of the vertices of the polyhedron. This is illustrated in Figure 1 for an arbitrary solid. 


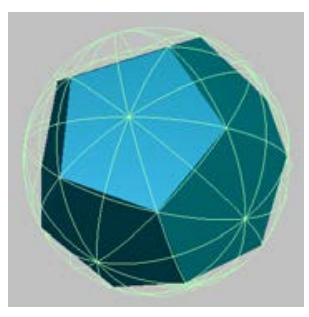

Figure 1. The spherical approximation for an arbitrary polyhedron.

Importantly, from Figure 1 it is evident that the volume of the sphere is larger than the volume of the original polyhedron. The ratio between the volume of the solid and circumscribed sphere is given by Equation (1) and depends on the particular geometry of the solid. Hence, the spherical approximation always results in an overestimation of the volume of a given particle. For nanoparticles, the volume is dependent on the density and the number of atoms; hence, an increase in volume leads to an apparent increase in the number of atoms (1).

$R_{\text {circumscribed }}=\frac{V_{\text {polyhedron }}}{V_{\text {circumscribed }}}$

SEM and TEM images typically provide a 2D plan view of the particles. An experimentalist then has to infer the 3D structure, which is difficult for polyhedra. This is because of a discrepancy between the sphericity and the circumscribed volume, which is quantified by the isoperimetric quotient, [21]

$I Q=36 \pi \frac{V^{2}}{S^{3}}$

where $V$ is the volume of the polyhedron and $S$ is its surface area. Only for a perfect sphere is $I Q=1$. Volumetric and sphericity data are shown in Figure 2 for the five platonic solids (tetrahedron, cube, octahedron, dodecahedron, and icosahedron), and the discrepancy between sphericity and the circumscribed volume is evident. The general trend is that with increasing number of vertices an increase in inscribed volume is observed. However, the increase in sphericity does not always correlate with the circumscribed volume as is the case for the icosahedron and dodecahedron. For quasi-spherical nanoparticles the spherical approximation is insufficient for accurate characterization and size determination.

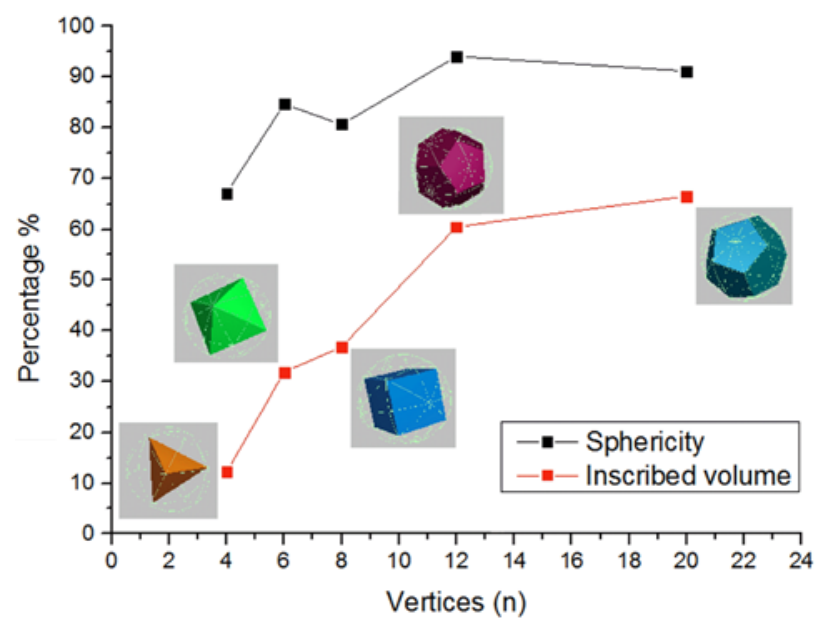

Figure 2. Relation between sphericity, circumscribed volume, and number of vertices for the platonic solids. 
In order to diminish the approximation error, three-dimensional volumetric information is required. In the case of nano-impacts one can obtain volumetric information from the charge passed during electrolysis, and then use it in conjunction with electron microscopy to size particles correctly. The validity of this idea is tested in the following sections for quasi-spherical silver nanoparticles.

\section{Experimental}

\subsection{Chemicals}

The chemicals used were of analytical grade and purchased from Sigma Aldrich unless stated otherwise. Quasi-spherical citrate-capped silver nanoparticles of nominal $50 \mathrm{~nm}$ radius were purchased from nanoComposix as an aqueous solution. For electrochemical experiments, $1.4 \mathrm{~mL}$ of stock solution was diluted to $10 \mathrm{~mL}$ with a $0.1 \mathrm{M}$ solution of $\mathrm{KCl}$.

\subsection{Characterization by SEM imaging}

Nanoparticle sizes and shapes were characterized by using high-resolution SEM imaging (SEM, LEO Gemini 1530, Zeiss). ImageJ software (W. S. Rasband, ImageJ, US National Institutes of Health, Bethesda, Maryland, USA, http://imagej. nih.gov/ij/ 1997-2014) was used for post-processing, particle analysis and area calculations.

\subsection{Electrochemical studies}

Electrochemical experiments were performed under thermostatic conditions $\left(25 \pm 1^{\circ} \mathrm{C}\right)$ by using a threeelectrode setup with an Autolab II potentiostat (Metrohm Autolab BV, Utrecht, Netherlands). A platinum mesh was used as the counter electrode and potentials were applied relative to a saturated calomel reference electrode (SCE). An aqueous $0.1 \mathrm{M} \mathrm{KCl}$ solution was prepared by using ultrapure water (Millipore, resistivity not less than $18.2 \mathrm{M} \Omega \mathrm{cm}$ at $25^{\circ} \mathrm{C}$ ). Previous work had shown that coulometric sizing was quantitative in chloride solution. [22] Anodic particle impacts were performed using a random array of microelectrodes (RAM) as a working electrode. A potential of $0.60 \mathrm{~V}$ vs SCE was chosen to ensure complete oxidation of the AgNPs. [23] A random assembly of microelectrodes [24] is an set of randomly dispersed carbon microfibers (approximately 3200 fibers) in non-conductive epoxy. Of the 3200 fibers approximately $20-40 \%$ are connected [24] and each of the fibers has a radius of 3.5 micrometers. The fibers are separated on average by 70 micrometers from each other. The ends of the fibers act as individual microdisks connected in parallel. The RAM was polished before the experiments using microcloth supplied by Buehler and $0.3 \mu \mathrm{M}$ alumina particles to ensure a clean and reproducible surface. For these studies, 20 chronoamperograms of $50 \mathrm{~s}$ duration were recorded at a potential of $0.60 \mathrm{~V}$ vs SCE and 256 spikes were recorded in total. The resulting data were analyzed using the software Signal Counter22 (developed by Dario Omanović of the Center for Marine and Environmental Research, Ruđer Bošković Institute, POB 180, 10002 Zagreb, Croatia) to identify spikes, perform baseline corrections and calculate the charge per given impact. The software Origin Pro 9.0 (Origin Lab Corporation) was used for data visualization and histogram analysis.

\subsection{Diffusional weighting}

According to the Stokes-Einstein Equation [25], larger nanoparticles have lower diffusion coefficients $(D)$ than smaller ones, leading to a lower probability of impacting a given microelectrode.[3]

$D=\frac{k_{\mathrm{B}} T}{6 \pi \eta r}$ 
Here $k_{\mathrm{B}}$ is the Boltzmann constant, $T$ is absolute temperature, $\eta$ is the solution viscosity, and $r$ is the radius of the particle. Evidently, differences in diffusion coefficients must be taken into account during size distribution analysis as the smaller particles have a higher probability of colliding with the electrode, causing the distribution to be skewed towards smaller nanoparticles. Diffusion-corrected weighting [26] can be applied to the data to compensate for this effect. The weighting is done by calculating the midpoint diffusion coefficient for each of the bars in the histogram and dividing the resulting diffusion coefficient by the diffusion coefficient of the smallest nanoparticles. In this way, individual bin counts can be adjusted.

\section{Results and Discussion}

The geometrical analysis described in the theoretical section was performed in three stages. First, the SEM images were analyzed and the spherical approximation was applied to obtain the size distribution. Second, the electrochemical sizing was carried out. Finally, the size distribution from the volumetric data was obtained.

\subsection{SEM image analysis}

The SEM images were analyzed using the ImageJ software. Automatic particle detection and particle area calculation was used. The average spherical radii $\left(r_{\text {average}}\right)$ were determined according to Equation (4):

$r_{\text {average }}=\sqrt{\frac{A}{\pi}}$

where $A$ was the projected area of the nanoparticle on the image. Analysis of 55 nanoparticles showed a size range of $45-60 \mathrm{~nm}$ in accordance with the manufacturer's specification. The size distribution shown in Figure 3 has the following characteristics: the mean radius is $50 \mathrm{~nm}$ and the standard deviation is $4 \mathrm{~nm}$. The SEM images clearly show that the particles are quasi-spherical and appear to have a hexagonal twodimensional projection as seen in Figure 3.

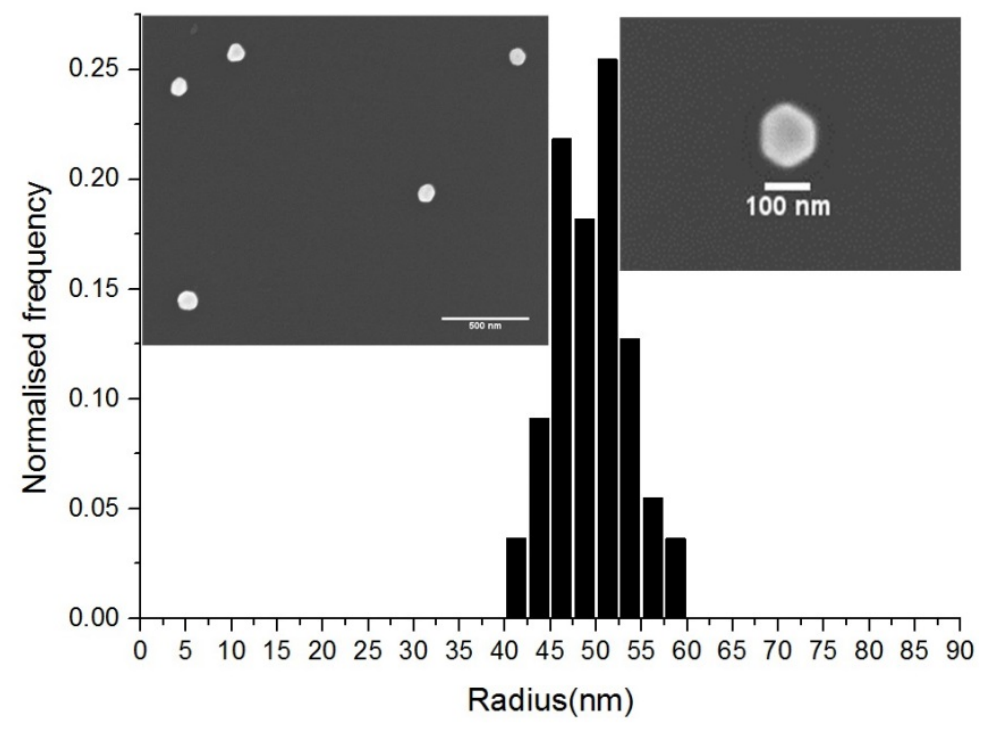

Figure 3. Size distribution of silver NPS as determined by SEM sizing. 


\subsection{Nano-impacts}

Previous nano-impact studies have used single microdisk electrodes. In the present study, we used a random assembly of microdisks (RAM) with a greater surface area (1000× area of 3.5 micrometer disk). The RAM allows detection of greater numbers of impacts, leading to higher accuracy of the particle size distribution. In addition, unlike micro-cylinder electrodes, [27] the RAMs are robust and can be re-polished many times. [24] A typical chronoamperogram is shown in Figure 4, where the spikes correspond to individual nanoparticle-RAM collisions. Spikes are not seen in the absence of NPs (Figure 4 grey transient). By integrating the area beneath a given spike, we can determine the charge of the colliding nanoparticle. If we assume that all AgNPs are spherical, and that full oxidation takes place, then we can calculate the associated Faradaic charge of a sphere with a mean radius obtained from SEM images ( $r=50$ $\mathrm{nm}$ ). We note that estimates of any capacitance charge assuming a double layer capacitance of $\approx 10$ $\mu \mathrm{Fcm}^{-2}$ correspond to less than $1 \%$ of the total charge. [28] Under a spherical approximation the relationship between charge passed and particle radius for $r=50 \mathrm{~nm}$ is given by Equation (5):

$Q_{\text {expected }}=\frac{8 F \pi \rho z r_{\mathrm{np}}^{3}}{3 A_{\mathrm{r}}} \approx 4.9 \times 10^{-12} \mathrm{C}$

where $F$ is the Faraday constant, $\rho$ is the density, $r_{\mathrm{np}}$ is the radius of the nanoparticle, $A_{\mathrm{r}}$ is the number of atoms in a nanoparticle, and $z=1$ is the charge per atom. From the integrated charge per spike and by assuming that the particle is spherical, we can calculate the corresponding radius. 256 impacts were analyzed and the raw radius distribution was obtained as a result of the above calculation. The mean experimental charge was approximately $2.5 \times 10^{-12} \mathrm{C}$, which is $\approx 51 \%$ of the charge calculated for $50 \mathrm{~nm}$ perfectly spherical NP from SEM images with the spherical approximation. The mean calculated nominal radius from impact experiments is $40 \mathrm{~nm}$, with a standard deviation of $10 \mathrm{~nm}$. The nano-impact experiments show consistently lower charge than anticipated for an idealized sphere with a $50 \mathrm{~nm}$ radius. One possible cause of the discrepancy could be the diffusion of the nanoparticles. As a result of the diffusion correction, the adjusted nominal mean radius would be $41 \mathrm{~nm}$ with a standard deviation of $10 \mathrm{~nm}$. This suggests that the difference in the diffusion of the particles alone cannot account for the discrepancies in the sizes obtained from SEM imaging and the nano-impacts.

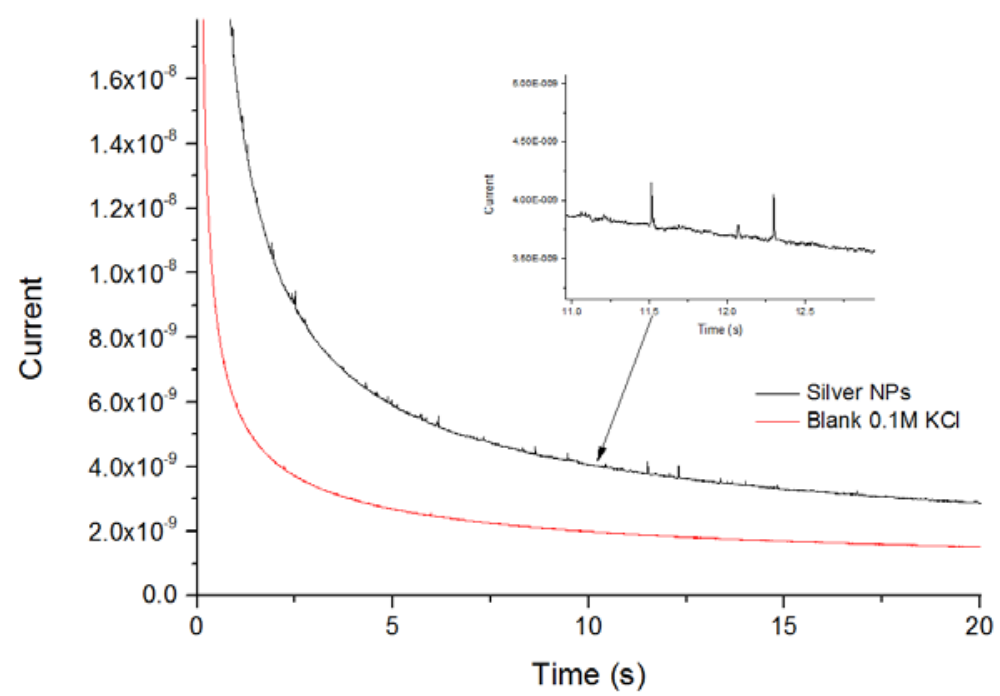

Figure 4. Chronoamperogram obtained from a RAM electrode with nanoparticles present (black), compared with chronoamperogram of blank (red). Note the absence of spikes. 


\subsection{Geometrical considerations}

The obtained size distribution from nano-impacts using the idealized spherical approximation is consistently lower compared with that from SEM images as shown in Figure 5. To account for this discrepancy, we present in the following section an explanation based on the quasi-spherical shape of the particles. From the SEM images shown in Figure 3, it is possible to introduce a simplified geometry more consistent with the images. A three-dimensional solid is required, which when viewed in two dimensions corresponds to an observed hexagonal shape. An icosahedron fits such requirements. It belongs to the set of platonic solids [29], having twelve vertices with twenty equilateral faces. This is shown in Figure 2. Platonic solids are ubiquitous in nature and there are well-attested examples of nanocubes [4] and nanotetrahedrons [30] and others. [31] An icosahedron is likely to lie on its face if the SEM grid surface is locally smooth; and hence, when viewed from above, it would appear as a hexagon as shown in Figure 3. Hence, icosahedron-shaped particles can account for the missing charge relative to the spherical approximation.

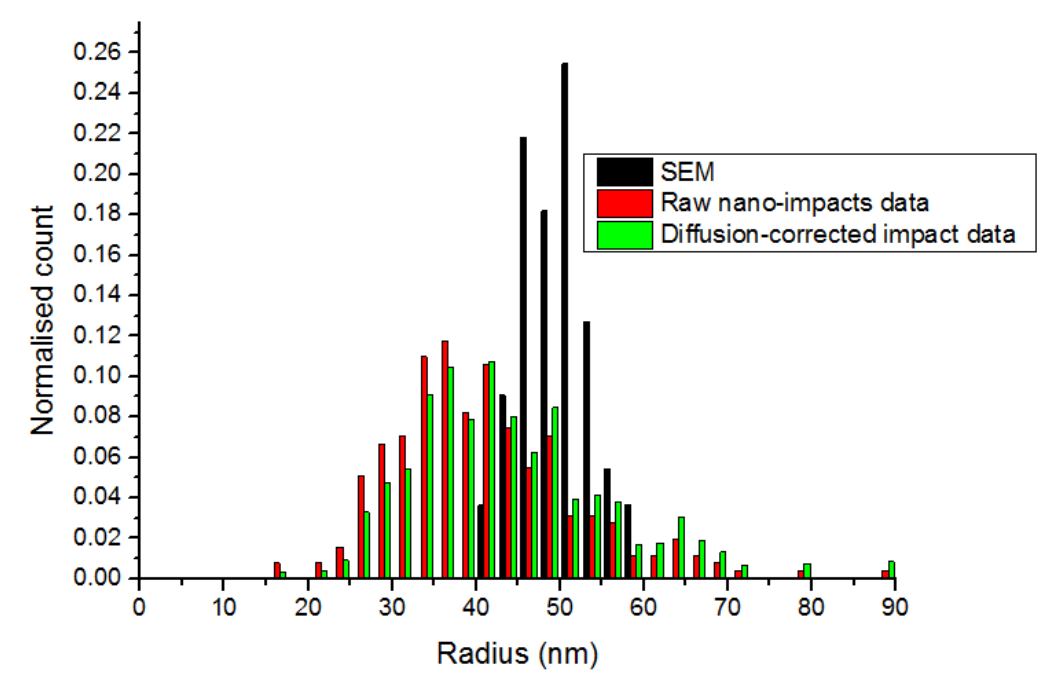

Figure 5. Comparison between size distributions obtained from SEM and nano-impacts sizing using the spherical approximation.

From the SEM images, we can find the length of the diagonal of the hexagon and find the edge length of an icosahedron. [32] As can be seen from Figure 2, the diagonal is equal to the diameter of the enclosing sphere. We can also express the edge length $\left(a_{\text {edge }}\right)$ in terms of the diagonal length $(d)$ [Equation (6)]. [6]

$a_{\text {edge }}=\frac{d}{\sqrt{\Phi^{2}+1}}$

where $\Phi$ is the golden ratio $\sim 1.61803399$. The volume of a icosahedron is given by Equations (7-9).

$$
\begin{aligned}
& V=\frac{5(3+\sqrt{5})}{12} a_{\text {edge }}^{3}=\frac{5(3+\sqrt{5})}{12}\left(\frac{d}{\sqrt{\Phi^{2}+1}}\right)^{3} \\
& d_{\text {icosahedron }}=2 r_{\text {sphere }}
\end{aligned}
$$


$V_{\text {circumscribed }}=\frac{V_{\text {icosahedron }}}{V_{\text {sphere }}} \approx 60.5 \%$

For an icosahedron, the circumscribed volume is $60.5 \%$ of a unit sphere. As a result, due to a reduction in the number of atoms, nano-impacts would yield the corresponding reduction in charge. Hence, for an icosahedron circumscribed by a sphere of radius $50 \mathrm{~nm}$, the corresponding charge would be almost $40 \%$ of that for a sphere. As a result, when converting charge distribution obtained from nano-impacts to radius distribution of the circumscribed sphere, we need to include a correction of $40 \%$, leading to a significant increase in the determined radius. Dividing the obtained charge for the impacts by 0.605 , we obtain the charge of the corresponding sphere and can calculate the corresponding radius according to Equation (5).

The surface area of an icosahedron is given by Equation (10):

$S_{\text {icosahedron }}=5 \times \sqrt{3} \times a_{\text {edge }}^{2}$

and hence, according to Equation (2), it has the highest sphericity of all the platonic solids at $93.9 \%$, but there is a significant difference in the inscribed volume relative to the circumscribed sphere. As a result, it appears almost spherical from two dimensional images, which can lead to significant sizing errors, if SEM imaging is used as the only sizing technique.

In light of the above considerations, the distributions become coincident within experimental error.

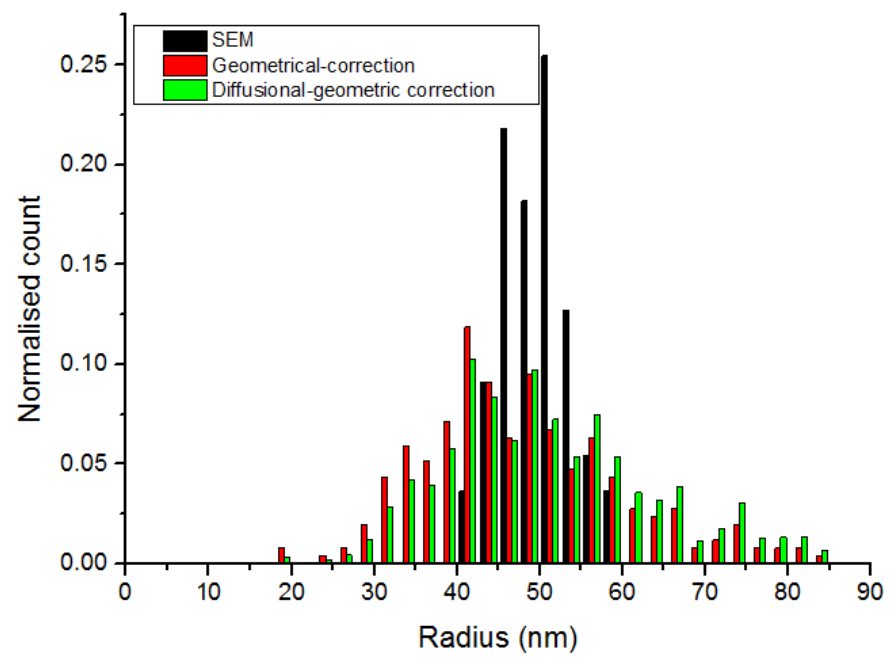

Figure 6. Normalized size distributions according to SEM imaging and nano-impacts with the applied geometric correction.

\section{Conclusion}

Used on its own, electron microscopy may be inadequate to quantify quasi-spherical particles, due to the inherent limitations of the two-dimensional view. Without the additional information provided by nano- 
impacts, the characterization is incomplete. A common method of describing nanoparticles is to use the radius of a corresponding sphere, but while this may be adequate in some cases, it can fail in others (for example, in the case of icosahedral particles). Thus, sizing by electron microscopy should ideally be complemented by a coulometric technique, such as that described in the present work. Overall, our results highlight the importance of the geometry of the quasi-spherical particles.

\section{Acknowledgements}

The research leading to these results has received funding from the European Research Council under the European Union's Seventh Framework Program (FP/2007-2013/ERC Grant Agreement no. [320403]). K.T. was supported by a Marie Curie Intra-European Fellowship within the Seventh European Community Framework Program.

\section{References}

1. A. Albanese, P. S. Tang, W. C. Chan, Annu. Rev. Biomed. Eng. 2012, 14, 1-16.

2. M. Notariannia, K. Vernona, A. Choua, M. Aljadab, J. Liua, N. Motta, Sol. Energy 2014, 106, $23-37$.

3. R. Mathaes, G. Winter, A. Besheer, J. Engert, Expert Opin. Drug Delivery 2014, 12, 1-12.

4. P. Guardia, R. Di Corato, L. Lartigue, C. Wilhelm, A. Espinosa, M. Garcia-Hernandez, F. Gazeau, L. Manna, T. Pellegrino, ACS Nano 2012, 6, 3080-3091.

5. D. Uzio, G. Berhault, Catal. Rev. 2010, 52, 106-131.

6. D. Su, Transmission Electron Microscopy Characterization of Nanomaterials, Springer, Heidelberg, 2014.

7. A. J. Kostera, U. Ziesea, A. J. Verkleija, A. H. Janssenb, J. de Graafb, J. W. Geusb, K. P. de Jong, Stud. Surf. Sci. Catal. 2000, 130, 329-334.

8. B. J. Berne, R. Pecora, Dynamic Light Scattering: With Applications to Chemistry, Biology, and Physics, Dover Publications, New York (USA), 2000.

9. S. Nath, C. Kaittanis, V. Ramachandran, N. S. Dalal, J. M. Perez, Chem. Mater. 2009, 21, 17611767.

10. J. K. Lim, D. X. Tan, F. Lanni, R. D. Tilton, S. A. Majetich, Magn. Magn. Mater. Dig. 2009, 321, 1557-1562.

11. V. Filipe, A. Hawe, W. Jiskoot, Pharm. Res. 2010, 27, 796-810.

12. M. Heyrovský, J. Jirkovský, Langmuir 1995, 11, 4288-4292.

13. Y. G. Zhou, N. V. Rees, R. G. Compton, Angew. Chem. Int. Ed. 2011, 50, 4219-4221; Angew. Chem. 2011, 123, 4305-4307.

14. Y. G. Zhou, E. J. E. Stuart, J. Pillay, S. Vilakazi, R. Tshikhudo, N. V. Rees, R. G. Compton, Chem. Phys. Lett. 2012, 551, 68-71.

15. K. Tschulik, B. Haddou, D. Omanović, N. V. Rees, R. G. Compton, Nano Res. 2013, 6, 836-841.

16. M. Pumera, ACS Nano 2014, 8, 7555-7558.

17. W. Cheng, X. F. Zhou, R. G. Compton, Angew. Chem. Int. Ed. 2013, 52, 12980-12982; Angew. Chem. 2013, 125, 13218-13220.

18. B.-K. Kim, A. Boika, J. Kim, J. E. Dick, A. J. Bard, J. Am. Chem. Soc. 2014, 136, 4849-4852.

19. N. A. Pangarov, V. Velinov, Electrochim. Acta 1968, 13, 1909-1914.

20. I. S. Yasnikov, Y. S. Nagornov, I. N. Protasova, E. E. Ivashko, E. E. Orozaliev, V. V. Dvoryadkin, A. V. Titov, Fundamental Research 2013, 4, 85-89.

21. G. Polya, Induction and Analogy in Mathematics, Princeton University Press, Princeton, NJ, 1954.

22. J. Ellison, K. Tschulik, E. J. E. Stuart, K. Jurksch, D. Omanovic, M. Uhlemann, A. Crossley, R. G. Compton, ChemistryOpen 2013, 2, 69-75.

23. E. J. E. Stuart, N. V. Rees, J. T. Cullen, R. G. Compton, Nanoscale 2013, 5, 174-177. 
24. S. Fletcher, M. D. Horne, Electrochem. Commun. 1999, 1, 502-512.

25. A. Einstein, Annalen der Physik 1905, 322, 549-560.

26. J. C. Lees, J. Ellison, C. Batchelor-McAuley, K. Tschulik, C. Damm, D. Omanovic, R. G. Compton, ChemPhysChem 2013, 14, 3895-3897.

27. J. Ellison, C. Batchelor-McAuley, K. Tschulik, R. G. Compton, Sensors Actuat. B 2014, 200, 47-52.

28. A. J. Bar, L. R. Faulkner, Electrochemical Methods: Fundamentals and Applications, John Wiley \& Sons 2001.

29. M. Atiyah, P. Sutcliffe, Milan J. Math. 2003, 71, no. 1, pp. 33-58

30. S. Gao, J. Yang, Z. Li, X. Jia, Y. Chen, J. Hazard. Mater. 2012, 211-212, 55-61.

31. F. Kim, S. Connor, H. Song, T. Kuykendall, P. Yang, Angew. Chem. Int. Ed. 2004, 43, 3673-3677; Angew. Chem. 2004, 116, 3759-3763.

32. K. J. M. MacLean, A Geometric Analysis of the Platonic Solids and Other Semi-Regular Polyhedra (Geometric Explorations), Loving Healing Press, Ann Arbor (USA), 2007. 